\title{
Some Fourier inequalities for orthogonal systems in Lorentz-Zygmund spaces
}

\section{G. Akishev ${ }^{1,2}$, L.E. Persson ${ }^{3 *}$ and A. Seger ${ }^{3}$}

\section{"Correspondence:}

larserik6pers@gmail.com

${ }^{3}$ Department of Computer Science

and Computational Engineering

Campus Narvik, The Artic University

of Norway, Narvik, Norway

Full list of author information is

available at the end of the article

\begin{abstract}
A number of classical inequalities and convergence results related to Fourier coefficients with respect to unbounded orthogonal systems are generalized and complemented. All results are given in the case of Lorentz-Zygmund spaces.
\end{abstract}

MSC: 42A16; 42B05; 26D15; 26D20; 46E30

Keywords: Inequalities; Fourier series; Fourier coefficients; Unbounded orthogonal systems; Lorentz-Zygmund spaces

\section{Introduction}

Let $q \in(1,+\infty), r \in(0,+\infty)$ and $\alpha \in \mathbb{R}$. Moreover, let $L_{q, r}(\log L)^{\alpha}$ denote the LorentzZygmund space, which consists of all measurable functions $f$ on $[0,1]$ such that

$$
\|f\|_{q, r, \alpha}:=\left\{\int_{0}^{1}\left(f^{*}(t)\right)^{r}(1+|\ln t|)^{\alpha r} \cdot t^{\frac{r}{q}-1} d t\right\}^{\frac{1}{r}}<+\infty,
$$

where $f^{*}$ is a nonincreasing rearrangement of the function $|f|$ (see e.g. [1]).

If $\alpha=0$, then the Lorentz-Zygmund space coincides with the Lorentz space: $L_{q_{1}, q_{2}}(\log L)^{\alpha}=L_{q_{1}, q_{2}}$. If $\alpha=0$ and $q_{1}=q_{2}=q$, then $L_{q_{1}, q_{2}}(\log L)^{\alpha}$ space coincides with the Lebesgue space $L_{q}[0,1]$ (see e.g. [2]) with the norm

$$
\|f\|_{q}:=\left(\int_{0}^{1}|f(x)|^{q} d x\right)^{\frac{1}{q}}, \quad 1 \leq q<+\infty .
$$

Moreover, $L_{\infty}[0,1]$ denotes the space, which consists of all measurable function on $[0,1]$ such that

$$
\|f\|_{\infty}:=\underset{x \in[0,1]}{\operatorname{ess} \sup }|f(x)|<\infty .
$$

We consider an orthogonal system $\left\{\varphi_{n}\right\}$ in $L_{2}[0,1]$ such that

$$
\left\|\varphi_{n}\right\|_{s} \leq M_{n}, \quad n \in \mathbb{N}
$$

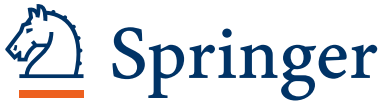

(c) The Author(s) 2019. This article is distributed under the terms of the Creative Commons Attribution 4.0 International License (http://creativecommons.org/licenses/by/4.0/), which permits unrestricted use, distribution, and reproduction in any medium, provided you give appropriate credit to the original author(s) and the source, provide a link to the Creative Commons license, and indicate if changes were made. 
and

$$
\mu_{n}=\sup \left\{\left\|\sum_{k=1}^{n} c_{k} \varphi_{k}\right\|_{s}: \sum_{k=1}^{n} c_{k}^{2}=1\right\}, \quad \rho_{n}=\left(\sum_{k=n}^{\infty}\left|a_{k}\right|^{2}\right)^{\frac{1}{2}},
$$

for some $s \in(2,+\infty]$. Here $M_{n} \uparrow$ and $M_{n} \geq 1$ (see [3], [4, p. 313]).

An orthonormal system $\left\{u_{n}\right\}$ is called uniformly bounded if there is a constant $M>0$ such that $\left\|u_{n}\right\|_{\infty} \leq M, \forall n \in N$. Note that any uniformly bounded system $\left\{u_{n}\right\}$ satisfies condition (1) but the reversed implication is false.

For one variable function Marcinkiewicz and Zygmund [4] proved the following theorems.

Theorem A (see [4]) Let the orthogonal system $\left\{\varphi_{n}\right\}$ satisfy the condition (1) and $2 \leq p<s$. If the real number sequence $\left\{a_{n}\right\}$ satisfies the condition

$$
\sum_{n=1}^{\infty}\left|a_{n}\right|^{p} M_{n}^{(p-2) \frac{s}{s-2}} n^{(p-2) \frac{s-1}{s-2}}<+\infty
$$

then the series

$$
\sum_{n=1}^{\infty} a_{n} \varphi_{n}(x)
$$

converges in $L_{p}$ to some function $f \in L_{p}[0,1]$ and

$$
\|f\|_{p} \leq C_{p, s}\left(\sum_{n=1}^{\infty}\left|a_{n}\right|^{p} M_{n}^{(p-2) \frac{s}{s-2}} n^{(p-2) \frac{s-1}{s-2}}\right)^{\frac{1}{p}}
$$

Theorem B (see [4]) Let the orthogonal system $\left\{\varphi_{n}\right\}$ satisfy the condition (1), and $\frac{s}{s-1}=$ $\mu<p \leq 2$. Then the Fourier coefficients $a_{n}(f)$ of the function $f \in L_{p}[0,1]$ with respect to the system $\left\{\varphi_{n}\right\}$ satisfy the inequality

$$
\left(\sum_{n=1}^{\infty}\left|a_{n}(f)\right|^{p} M_{n}^{(p-2) \frac{s}{s-2}} n^{(p-2) \frac{s-1}{s-2}}\right)^{\frac{1}{p}} \leq C_{p, s}\|f\|_{p} .
$$

Nowadays there are several generalizations of Theorems A and B for different spaces and systems (see e.g. [5-8] and the corresponding references).

Here we just mention that Flett [8] generalized this to the case of Lorentz spaces and that Maslov [5] proved generalizations of Theorem A and Theorem B in Orlicz spaces.

The problem concerning the summability of the Fourier coefficients by bounded orthonormal system with functions from some Lorentz spaces were investigated e.g. by Stein [9], Bochkarev [10], Kopezhanova and Persson [11] and Kopezhanova [12] (cf. also Persson [13]).

Moreover, Kolyada [6] proved the following improvement of Theorem A. 
Theorem C (see [6]) Let an orthogonal system $\left\{\varphi_{n}\right\}$ satisfy the condition (1), let the sequence $\left\{a_{n}\right\} \in l_{2}$ and $\rho_{n}=\left(\sum_{k=n}^{\infty}\left|a_{k}\right|^{2}\right)^{\frac{1}{2}}, 2<q<s \leq+\infty$. If

$$
\Lambda_{q}(a)=\left[\sum_{n=1}^{\infty} \mu_{n}^{\frac{(q-2) s}{s-2}}\left(\rho_{n}^{q}-\rho_{n+1}^{q}\right)\right]^{\frac{1}{q}}<+\infty
$$

then the series $\sum_{n=1}^{\infty} a_{n} \varphi_{n}(x)$ converges in the space $L_{q}$ to some function $f \in L_{q}$ and the following inequality holds: $\|f\|_{q} \leq C_{q, s} \Lambda_{q}(a)$.

This result was further generalized by Kirillov [7] as follows.

Theorem D (see [7]) If $2<q<s, r>0, \delta=\frac{r(q-2) s}{q(s-2)}$ and the sequence $\left\{a_{n}\right\} \in l_{2}$ satisfies the following condition:

$$
\Omega_{q, r}(a)=\left(\sum_{n=1}^{\infty}\left(\rho_{n}^{r}-\rho_{n+1}^{r}\right) \mu_{n}^{\delta}\right)^{\frac{1}{r}}<\infty \quad\left(\mu_{n} \equiv \mu_{n}^{(s)}, \quad \rho_{n}=\left(\sum_{k=n}^{\infty}\left|a_{k}\right|^{2}\right)^{\frac{1}{2}}\right),
$$

then the series $\sum_{n=1}^{\infty} a_{n} \varphi_{n}(x)$ converges in space $L_{2}[0,1]$ to some function $f$ and the inequality $\|f\|_{q, r} \leq C_{q, r, s} \Omega_{q, r}(a)$ holds. (Here $\mu_{n}$ and $\rho_{n}$ are defined by (2).)

The following well-known lemma is used in our proofs.

Lemma $\mathrm{E}$ Let $0<p<\infty$, and $\left\{a_{k}\right\}_{k=0}^{\infty}$ and $\left\{b_{k}\right\}_{k=0}^{\infty}$ are non-negative sequences.

(i) If

$$
\sum_{n=k}^{\infty} a_{n} \leq C a_{k}, \quad k=0,1,2, \ldots
$$

then

$$
\sum_{n=0}^{\infty} a_{n}\left(\sum_{k=0}^{n} b_{k}\right)^{p} \leq C p^{p} \sum_{n=0}^{\infty} a_{n} b_{n}^{p}
$$

(ii) If

$$
\sum_{n=0}^{k} a_{n} \leq C a_{k}, \quad k=0,1,2, \ldots,
$$

then

$$
\sum_{n=0}^{\infty} a_{n}\left(\sum_{k=n}^{\infty} b_{k}\right)^{p} \leq C p^{p} \sum_{n=0}^{\infty} a_{n} b_{n}^{p}
$$

where $C$ is a positive number independent of $n$.

In this paper we both generalize and complement the statements in Theorems A-D in various ways and always to the case with Lorentz-Zygmund spaces involved. In particular, in Sect. 2 such a generalization of Theorem D (and, thus, of Theorems A and C) is 
proved (see Theorem 2.1). In Sect. 3 such a complement of Theorem B to the case $q<2$ is given (see Theorem 3.1). Finally, in Sect. 4 we present and prove some further results for uniformly bounded systems and give some concluding remarks. In particular, we compare our results with some other recent research. For the reader's convenience we also include a proof of Lemma $\mathrm{E}$ in the Appendix.

\section{Generalization of Theorem D}

In this section we state and prove the following generalization of Theorem D.

Theorem 2.1 Let $2<q<s \leq+\infty, \alpha \in \mathbb{R}, r>0$ and $\delta=\frac{r s(q-2)}{q(s-2)}$. If $\left\{a_{n}\right\} \in l_{2}$ and

$$
\Omega_{q, r, \alpha}(a)=\left\{\sum_{n=1}^{\infty}\left(\rho_{n}^{r}-\rho_{n+1}^{r}\right) \mu_{n}^{\delta}\left(1+\frac{2 s}{s-2} \ln \mu_{n}\right)^{\alpha r}\right\}^{\frac{1}{r}}<+\infty,
$$

where $\rho_{n}$ and $\mu_{n}$ are defined by (2), then the series

$$
\sum_{n=1}^{\infty} a_{n} \varphi_{n}(x)
$$

with respect to an orthogonal system $\left\{\varphi_{n}\right\}_{n=1}^{\infty}$, which satisfies the condition (1), converges to some function $f \in L_{q, r}(\log L)^{\alpha}$ and $\|f\|_{q, r, \alpha} \leq C \Omega_{q, r, \alpha}$.

Corollary 2.2 For the case $\alpha=0$, Theorem 2.1 coincides with Theorem D.

Proof Since the sequence $\left\{\mu_{n}\right\}$ is increasing, let us define the sequence $\left\{v_{n}\right\}$ in the following way (see [7]):

$$
v_{1}=1, \quad v_{n+1}=\min \left\{k \in \mathbb{N}: \mu_{k} \geq 2 \mu_{v_{n}}\right\}, \quad n=1,2,3, \ldots
$$

Then $\mu_{v_{n+1}} \geq 2 \mu_{v_{n}}, \mu_{v_{n+1}-1}<2 \mu_{v_{n}}, n=1,2, \ldots$.

$$
\text { Let } t_{n}=\mu_{n}^{-\frac{2 s}{s-2}} \text {, }
$$

$$
\begin{aligned}
& u_{j}(x)=\sum_{k=v_{j}}^{\nu_{j+1}-1} a_{k} \varphi_{k}(x), \\
& S_{n}(x)=\sum_{k=1}^{n} u_{j}(x) \text { and } R_{n}(x)=f(x)-S_{n}(x) .
\end{aligned}
$$

Since $t_{n} \downarrow 0$ for $n \rightarrow+\infty$, by the property of nonincreasing rearrangement of the function (see [14, p. 83]), we get

$$
\begin{aligned}
\|f\|_{q, r, \alpha}^{r}= & \sum_{n=1}^{\infty} \int_{t_{n+1}}^{t_{n}}\left(f^{* *}(t)\right)^{r}(1+|\ln t|)^{\alpha r} t^{\frac{r}{q}-1} d t \\
\leq & C\left[\sum_{n=1}^{\infty} \int_{t_{n+1}}^{t_{n}}\left(S_{n}^{* *}(t)\right)^{r}(1+|\ln t|)^{\alpha r} t^{\frac{r}{q}-1} d t\right. \\
& \left.+\sum_{n=1}^{\infty} \int_{t_{n+1}}^{t_{n}}\left(R_{n}^{* *}(t)\right)^{r}(1+|\ln t|)^{\alpha r} t^{\frac{r}{q}-1} d t\right]:=C\left[I_{1}+I_{2}\right]
\end{aligned}
$$


and, moreover,

$$
S_{n}^{* *}(t) \leq \frac{1}{t} \sum_{j=1}^{n} \int_{0}^{t} u_{j}^{*}(y) d y
$$

By applying Hölder's inequality we obtain

$$
\int_{0}^{t} u_{j}^{*}(y) d y \leq t^{1-\frac{1}{s}} \rho_{v_{j}} \mu_{v_{j+1}-1}
$$

Therefore,

$$
S_{n}^{* *}(t) \leq t^{-\frac{1}{s}} \sum_{j=1}^{n} \rho_{v_{j}} \mu_{v_{j+1}-1}
$$

By using this estimate we find that

$$
\begin{aligned}
I_{1} & \leq \sum_{n=1}^{\infty} \int_{t_{n+1}}^{t_{n}}\left(\sum_{j=1}^{n} \rho_{v_{j}} \mu_{v_{j+1}-1}\right)^{r}(1+|\ln t|)^{\alpha r} t^{r\left(\frac{1}{q}-\frac{1}{s}\right)-1} d t \\
& \leq C \sum_{n=1}^{\infty}\left(\sum_{j=1}^{n} \rho_{v_{j}} \mu_{v_{j+1}-1}\right)^{r}\left(1+\left|\ln t_{n}\right|\right)^{\alpha r}\left(t_{n}^{r\left(\frac{1}{q}-\frac{1}{s}\right)}-t_{n+1}^{r\left(\frac{1}{q}-\frac{1}{s}\right)}\right) .
\end{aligned}
$$

Thus, by taking into account the definition of $t_{n}$, we can conclude that

$$
I_{1} \leq \sum_{n=1}^{\infty}\left(\sum_{j=1}^{n} \rho_{v_{j}} \mu_{v_{j+1}-1}\right)^{r}\left(1+\left|\frac{2 s}{s-2} \ln \mu_{v_{n}}\right|\right)^{\alpha r} \mu_{v_{n}}^{-r \frac{2(s-q)}{q(s-2)}} .
$$

Since for any $\varepsilon>0$ the function $t^{-\varepsilon} \ln t \downarrow 0$ for $t \rightarrow+\infty$, according to the definition of the numbers $v_{n}$, we see that

$$
\begin{aligned}
& \sum_{k=n}^{\infty}\left(1+\left|\frac{2 s}{s-2} \ln \mu_{v_{k}}\right|\right)^{\alpha r} \mu_{v_{k}}^{-r \frac{2(s-q)}{q(s-2)}} \\
& \quad \leq\left(\frac{1+\left|\frac{2 s}{s-2} \ln \mu_{v_{n}}\right|}{\mu_{v_{n}}^{\varepsilon}}\right)^{\alpha r} \mu_{v_{n}}^{-r\left(\frac{2(s-q)}{q(s-2)}-\varepsilon \alpha\right)} \sum_{k=n}^{\infty} 2^{-(k-n) r\left(\frac{2(s-q)}{q(s-2)}-\varepsilon \alpha\right)} .
\end{aligned}
$$

Now choose the number $\varepsilon$ such that $\frac{2(s-q)}{q(s-2)}-\varepsilon \alpha>0$. Then

$$
\sum_{k=n}^{\infty} 2^{-(k-n) r\left(\frac{2(s-q)}{q(s-2)}-\varepsilon \alpha\right)} \leq \sum_{l=0}^{\infty} 2^{-\operatorname{lr}\left(\frac{2(s-q)}{q(s-2)}-\varepsilon \alpha\right)}<+\infty
$$

Hence,

$$
\sum_{k=n}^{\infty}\left(1+\left|\frac{2 s}{s-2} \ln \mu_{v_{k}}\right|\right)^{\alpha r} \mu_{v_{k}}^{-r \frac{2(s-q)}{q(s-2)}} \leq C\left(1+\left|\frac{2 s}{s-2} \ln \mu_{v_{n}}\right|\right)^{\alpha r} \mu_{v_{n}}^{-r \frac{2(s-q)}{q(s-2)}} .
$$


Therefore, by Lemma E, we have

$$
\begin{aligned}
& \sum_{n=1}^{\infty}\left(\sum_{j=1}^{n} \rho_{v_{j}} \mu_{v_{j+1}-1}\right)^{r}\left(1+\left|\frac{2 s}{s-2} \ln \mu_{v_{n}}\right|\right)^{\alpha r} \mu_{v_{n}}^{-r \frac{2(s-q)}{q(s-2)}} \\
& \quad \leq C \sum_{n=1}^{\infty}\left(\rho_{v_{n}} \mu_{v_{n+1}-1}\right)^{r}\left(1+\left|\frac{2 s}{s-2} \ln \mu_{v_{n}}\right|\right)^{\alpha r} \mu_{v_{n}}^{-r \frac{2(s-q)}{q(s-2)}}
\end{aligned}
$$

Thus, from (6) it follows that

$$
I_{1} \leq C \sum_{n=1}^{\infty}\left(\rho_{v_{n}} \mu_{v_{n}}\right)^{r}\left(1+\left|\frac{2 s}{s-2} \ln \mu_{v_{n}}\right|\right)^{\alpha r} \mu_{v_{n}}^{\delta}
$$

where $\delta=r \frac{2(s-q)}{q(s-2)}$. Since $\rho_{n} \rightarrow 0$ for $n \rightarrow+\infty$, it yields $\rho_{v_{n}}^{r}=\sum_{k=n}^{\infty}\left(\rho_{v_{k}}^{r}-\rho_{v_{k+1}}^{r}\right)$. Therefore, by changing the order of summation, we get

$$
\sum_{n=1}^{\infty} \rho_{v_{n}}^{r}\left(1+\left|\frac{2 s}{s-2} \ln \mu_{v_{n}}\right|\right)^{\alpha r} \mu_{v_{n}}^{\delta}=\sum_{k=1}^{\infty}\left(\rho_{v_{k}}^{r}-\rho_{v_{k+1}}^{r}\right) \sum_{n=1}^{k}\left(1+\left|\frac{2 s}{s-2} \ln \mu_{v_{n}}\right|\right)^{\alpha r} \mu_{v_{n}}^{\delta} .
$$

Since $\delta>0$ and $\mu_{v_{n+1}} \geq 2 \mu_{v_{n}}$, we have $\sum_{n=1}^{k} \mu_{v_{n}}^{\delta} \leq C \mu_{v_{k}}^{\delta}$. Hence, by again using Lemma E, from (8) it follows that

$$
\sum_{n=1}^{\infty} \rho_{v_{n}}^{r}\left(1+\left|\frac{2 s}{s-2} \ln \mu_{v_{n}}\right|\right)^{\alpha r} \mu_{v_{n}}^{\delta} \leq C \sum_{k=1}^{\infty} \rho_{v_{k}}^{r}\left(1+\left|\frac{2 s}{s-2} \ln \mu_{v_{k}}\right|\right)^{\alpha r} \mu_{v_{k}}^{\delta} .
$$

By now combining inequalities (7) and (9) we obtain

$$
I_{1} \leq C \sum_{k=1}^{\infty} \rho_{v_{k}}^{r}\left(1+\left|\frac{2 s}{s-2} \ln \mu_{v_{k}}\right|\right)^{\alpha r} \mu_{v_{k}}^{\delta}
$$

Next we estimate $I_{2}$. By using Hölder's inequality we find that $R_{n}^{* *}(t) \leq C t^{-\frac{1}{2}}\left\|R_{n}\right\|_{2}$. Therefore,

$$
\begin{aligned}
I_{2} & \leq C \sum_{n=1}^{\infty}\left\|R_{n}\right\|_{2} \int_{t_{n+1}}^{t_{n}}(1+|\ln t|)^{\alpha r} t^{r\left(\frac{1}{q}-\frac{1}{2}\right)-1} d t \\
& \leq C \sum_{n=1}^{\infty}\left\|R_{n}\right\|_{2}\left(1+\left|\ln t_{n}\right|\right)^{\alpha r} \int_{t_{n+1}}^{t_{n}} t^{r\left(\frac{1}{q}-\frac{1}{2}\right)-1} d t \\
& \leq C \sum_{n=1}^{\infty} \rho_{v_{n+1}}^{r}\left(1+\left|\frac{2 s}{s-2} \ln \mu_{v_{n+1}}\right|\right)^{\alpha r} \mu_{v_{n+1}}^{\delta} .
\end{aligned}
$$

Next, by repeating the proof of Eq. (9) we obtain

$$
\sum_{n=1}^{\infty} \rho_{v_{n+1}}^{r}\left(1+\left|\frac{2 s}{s-2} \ln \mu_{v_{n+1}}\right|\right)^{\alpha r} \mu_{v_{n+1}}^{\delta} \leq C \sum_{k=1}^{\infty} \rho_{v_{k}}^{r}\left(1+\left|\frac{2 s}{s-2} \ln \mu_{v_{k}}\right|\right)^{\alpha r} \mu_{v_{k}}^{\delta} .
$$


By combining the inequalities (11) and (12) we have

$$
I_{2} \leq C \sum_{k=1}^{\infty} \rho_{v_{k}}^{r}\left(1+\left|\frac{2 s}{s-2} \ln \mu_{v_{k}}\right|\right)^{\alpha r} \mu_{v_{k}}^{\delta}
$$

Moreover, in view of inequalities (10) and (13), from (5) it follows that

$$
\|f\|_{q, r, \alpha}^{r} \leq C \sum_{k=1}^{\infty} \rho_{v_{k}}^{r}\left(1+\left|\frac{2 s}{s-2} \ln \mu_{v_{k}}\right|\right)^{\alpha r} \mu_{v_{k}}^{\delta}
$$

in the case $\alpha>0$. Since $\alpha>0$ and $\mu_{n} \uparrow$, we see that

$$
\begin{aligned}
& \sum_{n=1}^{\infty}\left(\rho_{n}^{r}-\rho_{n+1}^{r}\right) \mu_{n}^{\delta}\left(1+\frac{2 s}{s-2} \ln \mu_{n}\right)^{\alpha r} \\
& \quad=\sum_{k=1}^{\infty} \sum_{n=v_{k}}^{v_{k+1}-1}\left(\rho_{n}^{r}-\rho_{n+1}^{r}\right) \mu_{n}^{\delta}\left(1+\frac{2 s}{s-2} \ln \mu_{n}\right)^{\alpha r} \\
& \geq \sum_{k=1}^{\infty} \mu_{v_{k}}^{\delta}\left(1+\frac{2 s}{s-2} \ln \mu_{v_{k}}\right)^{\alpha r} \sum_{n=v_{k}}^{v_{k+1}-1}\left(\rho_{n}^{r}-\rho_{n+1}^{r}\right) \\
& =\sum_{k=1}^{\infty} \mu_{v_{k}}^{\delta}\left(1+\frac{2 s}{s-2} \ln \mu_{v_{k}}\right)^{\alpha r}\left(\rho_{v_{k}}^{r}-\rho_{v_{k+1}}^{r}\right) .
\end{aligned}
$$

Hence, from the inequality (14) it follows that

$$
\|f\|_{q, r, \alpha}^{r} \leq C \sum_{n=1}^{\infty}\left(\rho_{n}^{r}-\rho_{n+1}^{r}\right) \mu_{n}^{\delta}\left(1+\frac{2 s}{s-2} \ln \mu_{n}\right)^{\alpha r}
$$

in the case $\alpha>0$.

Let $\alpha<0$. Then, for any number $\varepsilon>0$, the function $y^{\varepsilon}(1+\ln y)^{r \alpha}$ increases on $(1, \infty)$. Therefore, by taking into account that $\mu_{n} \uparrow$, we obtain

$$
\begin{aligned}
& \sum_{n=1}^{\infty}\left(\rho_{n}^{r}-\rho_{n+1}^{r}\right) \mu_{n}^{\delta}\left(1+\frac{2 s}{s-2} \ln \mu_{n}\right)^{\alpha r} \\
& \quad=\sum_{k=1}^{\infty} \sum_{n=v_{k}}^{v_{k+1}-1}\left(\rho_{n}^{r}-\rho_{n+1}^{r}\right) \mu_{n}^{\delta}\left(1+\frac{2 s}{s-2} \ln \mu_{n}\right)^{\alpha r} \\
& \quad \geq \sum_{k=1}^{\infty} \mu_{v_{k}}^{\varepsilon}\left(1+\frac{2 s}{s-2} \ln \mu_{v_{k}}\right)^{\alpha r} \sum_{n=v_{k}}^{v_{k+1}-1}\left(\rho_{n}^{r}-\rho_{n+1}^{r}\right) \mu_{n}^{\delta-\varepsilon} .
\end{aligned}
$$

Choose $\varepsilon>0$ such that $\delta-\varepsilon>0$. Since $\mu_{n}^{\delta-\varepsilon} \uparrow$, according to the inequality (16), we have

$$
\sum_{n=1}^{\infty}\left(\rho_{n}^{r}-\rho_{n+1}^{r}\right) \mu_{n}^{\delta}\left(1+\frac{2 s}{s-2} \ln \mu_{n}\right)^{\alpha r} \geq \sum_{k=1}^{\infty} \mu_{v_{k}}^{\delta}\left(1+\frac{2 s}{s-2} \ln \mu_{v_{k}}\right)^{\alpha r} \sum_{n=v_{k}}^{\nu_{k+1}-1}\left(\rho_{n}^{r}-\rho_{n+1}^{r}\right)
$$

in the case $\alpha<0$. Therefore (15) holds also for case $\alpha<0$ and the proof is complete. 
Corollary 2.3 Let $\left\{\varphi_{n}\right\}_{n=1}^{\infty}$ be an uniformly bounded orthogonal system and let $2<q<+\infty$, $\alpha \in \mathbb{R}$ and $r>0$.

If

$$
\Omega_{q, r}(a)=\left(\sum_{n=1}^{\infty}\left(\rho_{n}^{r}-\rho_{n+1}^{r}\right) n^{\frac{r(q-2)}{2 q}}(1+\ln n)^{\alpha r}\right)^{\frac{1}{r}}<\infty
$$

where $\rho_{n}$ are defined by (2), then the series $\sum_{n=1}^{\infty} a_{n} \varphi_{n}(x)$ converges to some function $f \in$ $L_{q, r}(\log L)^{\alpha}$ and $\|f\|_{q, r, \alpha} \leq C \cdot \Omega_{q, r, \alpha}$.

Proof Since $\left\{\varphi_{n}\right\}_{n=1}^{\infty}$ is an uniformly bounded orthogonal system, we have $s=+\infty$. Therefore

$$
\lim _{s \rightarrow+\infty} \frac{r s(q-2)}{q(s-2)}=\frac{r(q-2)}{q} .
$$

Now, given that $M_{n} \leq M, \mu_{n} \leq \sqrt{n} M, n \in \mathbb{N}$, we have

$$
\sum_{n=1}^{\infty}\left(\rho_{n}^{r}-\rho_{n+1}^{r}\right) \mu_{n}^{\delta}\left(1+\frac{2 s}{s-2} \ln \mu_{n}\right)^{\alpha r} \leq C \sum_{n=1}^{\infty}\left(\rho_{n}^{r}-\rho_{n+1}^{r}\right) n^{\frac{r(q-2)}{2 q}}(1+\ln n)^{\alpha r}
$$

if $\alpha \geq 0$.

If $\alpha<0$, then we choose a number $\varepsilon$ such that $0<\varepsilon<\frac{(q-2)}{q}$. Then, by considering the function $(1+\ln t)^{\alpha} t^{\varepsilon} \uparrow$ on $[1,+\infty)$, we can verify that the inequality (17) holds also for $\alpha<0$. Consequently, by Theorem 2.1, the statement is true.

\section{A complement of Theorem B. The case $q<2$}

In this section we prove a result which was formulated but not proven in [15]. It may be regarded as a complement of Theorem B relevant for a more general situation.

Theorem 3.1 Let $s \in(2,+\infty], \frac{s}{s-1}<q<2, r>1, \alpha \in \mathbb{R}$ and $\delta=\frac{r(q-2) s}{q(s-2)}$. If $f \in L_{q, r}(\log L)^{\alpha}$, then the inequality

$$
\left[\sum_{n=1}^{\infty}\left(\sum_{k=v_{n}}^{v_{n+1}-1} a_{k}^{2}(f)\right)^{\frac{r}{2}}\left(1+\log \mu_{v_{n}}\right)^{r \alpha} \mu_{v_{n}}^{\delta}\right]^{\frac{1}{r}} \leq C\|f\|_{q, r, \alpha}
$$

holds, where $\mu_{v_{n}}$ are defined by (2) and $a_{n}(f)$ denote the Fourier coefficients off with respect to an orthogonal system $\left\{\varphi_{n}\right\}_{n=1}^{\infty}$ satisfying condition (1).

Remark 3.2 Theorem 3.1 was formulated, but not proved, in [15]. Here we present the details of the proof.

Proof Choose an increasing sequence $\left\{v_{n}\right\}$ of natural numbers such that $v_{1}=1, v_{n+1}=$ $\min \left\{k: \mu_{k} \geq 2 \mu_{v_{n}}\right\}, n=1,2,3, \ldots$. Then $\mu_{v_{n+1}} \geq 2 \mu_{v_{n}}, \mu_{v_{n+1}-1}<2 \mu_{v_{n}}$. Since the system 
$\left\{\varphi_{n}\right\}$ is orthogonal we have

$$
\left|\int_{0}^{1} f(x) g(x) d x\right|=\left|\sum_{k=1}^{\infty} a_{k}(f) b_{k}(g)\right|
$$

for any function $g \in L_{q^{\prime}, r^{\prime}}(\log L)^{-\alpha}, \frac{1}{r}+\frac{1}{r^{\prime}}=1$ and $\frac{1}{q}+\frac{1}{q^{\prime}}=1$. Let

$$
\begin{aligned}
b_{k}:= & {\left[\sum_{n=1}^{\infty}\left(\sum_{k=v_{n}}^{v_{n+1}-1} a_{k}^{2}(f)\right)^{\frac{r}{2}}\left(1+\log \mu_{v_{n}}\right)^{r \alpha} \mu_{v_{n}}^{\delta}\right]^{-\frac{1}{r^{\prime}}} } \\
& \times\left(\sum_{k=v_{n}}^{v_{n+1}-1}\left|a_{k}(f)\right|^{2}\right)^{\frac{r-2}{2}}\left(1+\log \mu_{v_{n}}\right)^{r \alpha} \mu_{v_{n}}^{\delta} a_{k}(f)
\end{aligned}
$$

for $k=v_{n}, \ldots, v_{n+1}-1, n=1,2, \ldots$, and consider a function $g \in L_{q^{\prime}, r^{\prime}}(\log L)^{-2}$ with Fourier coefficients $b_{k}(g)=b_{k}$. Then

$$
\begin{aligned}
\left|\int_{0}^{1} f(x) g(x) d x\right| & =\left|\sum_{n=1}^{\infty} \sum_{k=v_{n}}^{v_{n+1}-1} a_{k}(f) b_{k}(g)\right| \\
& =\left[\sum_{n=1}^{\infty}\left(\sum_{k=v_{n}}^{v_{n+1}-1} a_{k}^{2}(f)\right)^{\frac{r}{2}}\left(1+\log \mu_{v_{n}}\right)^{r \alpha} \mu_{v_{n}}^{\delta}\right]^{\frac{1}{r}} .
\end{aligned}
$$

Taking into account that $r r^{\prime}=r+r^{\prime}$, by Theorem 2.1 and (18), we have

$$
\begin{aligned}
\|g\|_{q^{\prime}, r^{\prime},-\alpha} \leq & C\left\{\sum_{n=1}^{\infty}\left(\sum_{k=v_{n}}^{v_{n+1}-1} b_{k}^{2}(g)\right)^{\frac{r^{\prime}}{2}}\left(1+\log \mu_{v_{n}}\right)^{-r^{\prime} \alpha} \mu_{v_{n}}^{\frac{s\left(q^{\prime}-2\right)}{(s-2) q^{\prime}} \cdot r^{\prime}}\right\}^{\frac{1}{r^{\prime}}} \\
= & C\left[\sum_{n=1}^{\infty}\left(\sum_{k=v_{n}}^{v_{n+1}-1} a_{k}^{2}(f)\right)^{\frac{r}{2}}\left(1+\log \mu_{v_{n}}\right)^{r \alpha} \mu_{v_{n}}^{\delta}\right]^{-\frac{1}{r^{\prime}}} \\
& \times\left\{\sum_{n=1}^{\infty}\left(\sum_{k=v_{n}}^{v_{n+1}-1} a_{k}^{2}(f)\right)^{\frac{r^{\prime}}{2}}\left(\sum_{k=v_{n}}^{v_{n+1}-1} a_{k}^{2}(f)\right)^{\frac{r^{\prime}}{2}(r-2)}\right. \\
& \left.\times\left(1+\log \mu_{v_{n}}\right)^{-r^{\prime} \alpha} \mu_{v_{n}}^{\frac{s\left(q^{\prime}-2\right) q^{\prime}}{(s) \cdot r^{\prime}}}\left(1+\log \mu_{v_{n}}\right)^{r r^{\prime} \alpha} \mu_{v_{n}}^{\frac{s(q-2)}{(s-2)} r r^{\prime}}\right\}^{\frac{1}{r^{\prime}}} \\
= & C\left[\sum_{n=1}^{\infty}\left(\sum_{k=v_{n}}^{v_{n+1}-1} a_{k}^{2}(f)\right)^{\frac{r}{2}}\left(1+\log \mu_{v_{n}}\right)^{r \alpha} \mu_{v_{n}}^{\delta}\right]^{-\frac{1}{r^{\prime}}} \\
& \times\left[\sum_{n=1}^{\infty}\left(\sum_{k=v_{n}}^{v_{n+1}-1} a_{k}^{2}(f)\right)^{\frac{r}{2}}\left(1+\log \mu_{v_{n}}\right)^{r \alpha} \mu_{v_{n}}^{\delta}\right]^{\frac{1}{r^{\prime}}}=C .
\end{aligned}
$$


Thus, the function $g_{0}:=C^{-1} g \in L_{q^{\prime}, r^{\prime}}(\log L)^{-\alpha}$ and $\left\|g_{0}\right\|_{q^{\prime}, r^{\prime},-\alpha} \leq 1$. Next, by the property of the norm in the Lorentz-Zygmund space and using equality (19), we get

$$
\begin{aligned}
\|f\|_{q, r, \alpha} & \asymp \sup _{\substack{g \in L_{q^{\prime}, r^{\prime}}(\log L)^{-\alpha} \\
\|g\|_{q^{\prime}, r^{\prime},-\alpha} \leq 1}}\left|\int_{0}^{1} f(x) g(x) d x\right| \geq\left|\int_{0}^{1} f(x) g_{0}(x) d x\right| \\
& =C^{-1}\left[\sum_{n=1}^{\infty}\left(\sum_{k=v_{n}}^{v_{n+1}-1} a_{k}^{2}(f)\right)^{\frac{r}{2}} \mu_{v_{n}}^{\delta}\right]^{\frac{1}{r}} .
\end{aligned}
$$

The proof is complete.

\section{Further results and concluding remarks}

In this section we first prove some results which are closely related to but not covered by the results in the previous sections (Propositions 4.1 and 4.2). After that, we present some results of a similar kind (see $[11,12]$ and Theorem F) and in remarks we point out how these results can be compared with our results in some special cases when such a comparison is possible.

Proposition 4.1 Let $\left\{\varphi_{n}\right\}_{n=1}^{\infty}$ be an uniformly bounded orthogonal system and $2<q<+\infty$, $\alpha \in \mathbb{R}$ and $r>1$. If

$$
\Omega_{q, r, \alpha}(a)=\left(\sum_{n=1}^{\infty}\left|a_{n}\right|^{r} n^{r\left(1-\frac{1}{q}-\frac{1}{r}\right)}(1+\ln n)^{\alpha r}\right)^{\frac{1}{r}}<\infty
$$

then the series $\sum_{n=1}^{\infty} a_{n} \varphi_{n}(x)$ converges to some function $f \in L_{q, r}(\log L)^{\alpha}$ and $\|f\|_{q, r, \alpha} \leq$ $C \Omega_{q, r, \alpha}(a)$.

Proof Since $\rho_{n} \downarrow 0$ when $n \rightarrow+\infty$, we can choose numbers $n_{1}=1$,

$$
n_{k+1}=\min \left\{n \in \mathbb{N}: \rho_{n_{k+1}} \leq \frac{1}{2} \rho_{n_{k}}\right\}, \quad k=1,2 \ldots
$$

Therefore, if $\alpha \geq 0$, it yields

$$
\sum_{n=1}^{\infty}\left(\rho_{n}^{r}-\rho_{n+1}^{r}\right) n^{\frac{r(q-2)}{2 q}}(1+\ln n)^{\alpha r}=\sum_{k=2}^{\infty}\left(n_{k}-1\right)^{\frac{r(q-2)}{2 q}}\left(1+\ln n_{k}\right)^{\alpha r}\left(\rho_{n_{k-1}}^{r}-\rho_{n_{k}}^{r}\right)
$$

For any numbers $k=2,3, \ldots$, the following inequality holds:

$$
\rho_{n_{k-1}}^{r}-\rho_{n_{k}}^{r} \leq \rho_{n_{k-1}}^{r} \leq 2^{r}\left(\rho_{n_{k}-1}^{2}\right)^{\frac{r}{2}} .
$$

Since $\rho_{n_{k+1}} \leq \frac{1}{2} \rho_{n_{k}} \leq \frac{1}{2} \rho_{n_{k}-1}$, we have

$$
\rho_{n_{k}-1}^{2}-\rho_{n_{k+1}}^{2} \geq \rho_{n_{k}-1}^{2}-\left(\frac{1}{2} \rho_{n_{k}-1}\right)^{2}=\frac{3}{4} \rho_{n_{k}-1}^{2} .
$$


By using (21) and (22), we can obtain the following inequality:

$$
\rho_{n_{k-1}}^{r}-\rho_{n_{k}}^{r} \leq 2^{r}\left(\frac{4}{3}\right)^{\frac{r}{2}}\left(\sum_{n=n_{k}-1}^{n_{k+1}-1}\left|a_{n}\right|^{2}\right)^{\frac{r}{2}}
$$

Therefore, from (20) it follows that

$$
\sum_{n=1}^{\infty}\left(\rho_{n}^{r}-\rho_{n+1}^{r}\right) n^{\frac{r(q-2)}{2 q}}(1+\ln n)^{\alpha r} \leq C_{r} \sum_{k=2}^{\infty}\left(n_{k}-1\right)^{\frac{r(q-2)}{2 q}}\left(1+\ln n_{k}\right)^{\alpha r}\left(\sum_{n=n_{k}-1}^{n_{k+1}-1}\left|a_{n}\right|^{2}\right)^{\frac{r}{2}}
$$

when $\alpha \geq 0$.

If $\alpha<0$, then we can choose a number $\varepsilon$ which satisfies $0<\varepsilon<\frac{q-2}{2 q}$. We note that $(1+$ $\ln n)^{\alpha} n^{\varepsilon} \uparrow$ and we obtain the following inequality:

$$
\begin{aligned}
& \sum_{n=n_{k-1}}^{n_{k}-1}\left(\rho_{n}^{r}-\rho_{n+1}^{r}\right) n^{\frac{r(q-2)}{2 q}}(1+\ln n)^{\alpha r} \\
& \quad=\sum_{n=n_{k-1}}^{n_{k}-1}\left(\rho_{n}^{r}-\rho_{n+1}^{r}\right) n^{\frac{r(q-2)}{2 q}-r \varepsilon}\left((1+\ln n)^{\alpha} n^{\varepsilon}\right)^{r} \\
& \leq\left(n_{k}-1\right)^{\frac{r(q-2)}{2 q}-r \varepsilon}\left(\left(1+\ln \left(n_{k}-1\right)\right)^{\alpha}\left(n_{k}-1\right)^{\varepsilon}\right)^{r} \sum_{n=n_{k-1}}^{n_{k}-1}\left(\rho_{n}^{r}-\rho_{n+1}^{r}\right) \\
& =\left(n_{k}-1\right)^{\frac{r(q-2)}{2 q}}\left(1+\ln \left(n_{k}-1\right)\right)^{\alpha r}\left(\rho_{n_{k-1}}^{r}-\rho_{n_{k}}^{r}\right) .
\end{aligned}
$$

By now combining the inequalities (20), (23) and (25), we conclude that (24) holds also for the case $\alpha<0$.

If $r>2$, then, by using Hölder's inequality with $\theta=\frac{r}{2}, \frac{1}{\theta}+\frac{1}{\theta^{\prime}}=1$, we obtain

$$
\sum_{n=n_{k}-1}^{n_{k+1}-1}\left|a_{n}\right|^{2} \leq\left(\sum_{n=n_{k}-1}^{n_{k+1}-1}\left|a_{n}\right|^{r} n^{r\left(1-\frac{1}{q}\right)-1}\right)^{\frac{2}{r}}\left(\sum_{n=n_{k}-1}^{n_{k+1}-1} n^{\theta^{\prime}\left(\frac{1}{\theta}-2\left(1-\frac{1}{q}\right)\right)}\right)^{\frac{1}{\theta^{\prime}}}
$$

Since $2<q$, we have $1+\theta^{\prime}\left(\frac{1}{\theta}-2\left(1-\frac{1}{q}\right)\right)=\theta^{\prime}\left(\frac{2}{q}-1\right)<0$. Therefore,

$$
\begin{aligned}
\sum_{n=n_{k}-1}^{n_{k+1}-1} n^{\theta^{\prime}\left(\frac{1}{\theta}-2\left(1-\frac{1}{q}\right)\right)} & \leq C_{r, q} \int_{n_{k}-1}^{n_{k+1}} t^{\theta^{\prime}\left(\frac{1}{\theta}-2\left(1-\frac{1}{q}\right)\right)} d t \\
& \leq \frac{C_{r, q}}{\theta^{\prime}\left(2\left(1-\frac{1}{q}\right)-\frac{1}{\theta}\right)-1}\left(n_{k}-1\right)^{1+\theta^{\prime}\left(\frac{1}{\theta}-2\left(1-\frac{1}{q}\right)\right)}
\end{aligned}
$$

for $k=2,3, \ldots$. From inequalities (26) and (27), we can derive the following inequality:

$$
\sum_{n=n_{k}-1}^{n_{k+1}-1}\left|a_{n}\right|^{2} \leq C\left(n_{k}-1\right)^{\frac{1}{\theta^{\prime}}+\frac{1}{\theta}-2\left(1-\frac{1}{q}\right)}\left(\sum_{n=n_{k}-1}^{n_{k+1}-1}\left|a_{n}\right|^{r} n^{r\left(1-\frac{1}{q}\right)-1}\right)^{\frac{2}{r}}
$$

for $k=2,3, \ldots$, in the case of $2<r<\infty$. 
Now, by combining (26) and (28), we obtain the following inequality:

$$
\begin{aligned}
& \sum_{n=1}^{\infty}\left(\rho_{n}^{r}-\rho_{n+1}^{r}\right) n^{\frac{r(q-2)}{2 q}}(1+\ln n)^{\alpha r} \\
& \quad \leq \sum_{k=2}^{\infty}\left(n_{k}-1\right)^{\frac{r(q-2)}{2 q}}\left(1+\ln n_{k}\right)^{\alpha r}\left(n_{k}-1\right)^{\frac{r}{2}\left(1-2\left(1-\frac{1}{q}\right)\right)} \sum_{n=n_{k}-1}^{n_{k+1}-1}\left|a_{n}\right|^{r} n^{r\left(1-\frac{1}{q}\right)-1}
\end{aligned}
$$

in the case of $2<r<\infty, 0<\alpha<\infty$.

Since

$$
\frac{r(q-2)}{2 q}+\frac{r}{2}\left(1-2\left(1-\frac{1}{q}\right)\right)=0
$$

it follows from (29) that

$$
\sum_{n=1}^{\infty}\left(\rho_{n}^{r}-\rho_{n+1}^{r}\right) n^{\frac{r(q-2)}{2 q}}(1+\ln n)^{\alpha r} \leq C \sum_{k=2}^{\infty} \sum_{n=n_{k}-1}^{n_{k+1}-1}\left|a_{n}\right|^{r} n^{r\left(1-\frac{1}{q}\right)-1}(1+\ln n)^{\alpha r}
$$

in the case $2<r<\infty, 0<\alpha<\infty$.

Furthermore,

$$
\begin{aligned}
& \sum_{k=2}^{\infty} \sum_{n=n_{k}-1}^{n_{k+1}-1}\left|a_{n}\right|^{r} n^{r\left(1-\frac{1}{q}\right)-1}(1+\ln n)^{\alpha r} \\
& \quad \leq \sum_{k=2}^{\infty} \sum_{n=n_{k-1}}^{n_{k}-1}\left|a_{n}\right|^{r} n^{r\left(1-\frac{1}{q}\right)-1}(1+\ln n)^{\alpha r}+\sum_{k=2}^{\infty} \sum_{n=n_{k}}^{n_{k+1}-1}\left|a_{n}\right|^{r} n^{r\left(1-\frac{1}{q}\right)-1}(1+\ln n)^{\alpha r} \\
& \quad \leq 2 \sum_{n=1}^{\infty}\left|a_{n}\right|^{r} n^{r\left(1-\frac{1}{q}\right)-1}(1+\ln n)^{\alpha r}
\end{aligned}
$$

in the case $2<r<\infty, 0<\alpha<\infty$.

If $\alpha<0$, then we choose a number $\varepsilon$ which satisfies $0<\varepsilon<\frac{q-2}{2 q}$. By using the Hölder inequality, we obtain $\left(\theta=\frac{r}{2}, \frac{1}{\theta}+\frac{1}{\theta^{\prime}}=1\right)$

$$
\sum_{n=n_{k}-1}^{n_{k+1}-1}\left|a_{n}\right|^{2} \leq\left(\sum_{n=n_{k}-1}^{n_{k+1}-1}\left|a_{n}\right|^{r} n^{r\left(1-\frac{1}{q}\right)-\varepsilon \theta-1}\right)^{\frac{2}{r}}\left(\sum_{n=n_{k}-1}^{n_{k+1}-1} n^{\theta^{\prime}\left(\frac{1}{\theta}-2\left(1-\frac{1}{q}\right)+\varepsilon\right)}\right)^{\frac{1}{\theta^{\prime}}} .
$$

According to the choice of the number $\varepsilon$ it shows that

$$
1+\theta^{\prime}\left(\frac{1}{\theta}-2\left(1-\frac{1}{q}\right)+\varepsilon\right)=\theta^{\prime}\left(\frac{2}{q}-1+\varepsilon\right)<0 .
$$

Therefore (as in the case of $\alpha>0$ ) we obtain the following inequality:

$$
\begin{aligned}
\sum_{n=n_{k}-1}^{n_{k+1}-1} n^{\theta^{\prime}\left(\frac{1}{\theta}-2\left(1-\frac{1}{q}\right)+\varepsilon\right)} & \leq C_{r, q} \int_{n_{k}-1}^{n_{k+1}} t^{\theta^{\prime}\left(\frac{1}{\theta}-2\left(1-\frac{1}{q}\right)+\varepsilon\right)} d t \\
& \leq \frac{C_{r, q}}{\theta^{\prime}\left(2\left(1-\frac{1}{q}\right)-1-\varepsilon\right)}\left(n_{k}-1\right)^{1+\theta^{\prime}\left(\frac{1}{\theta}-2\left(1-\frac{1}{q}\right)+\varepsilon\right)}
\end{aligned}
$$


for $k=2,3, \ldots$ Thus, in view of (32) and (33), the following inequality holds:

$$
\sum_{n=n_{k}-1}^{n_{k+1}-1}\left|a_{n}\right|^{2} \leq C\left(n_{k}-1\right)^{1+\theta^{\prime}\left(\frac{1}{\theta}-2\left(1-\frac{1}{q}\right)+\varepsilon\right)}\left(\sum_{n=n_{k}-1}^{n_{k+1}-1}\left|a_{n}\right|^{r} n^{r\left(1-\frac{1}{q}\right)-\varepsilon \theta-1}\right)^{\frac{2}{r}}
$$

for the case of $2<r<\infty, \alpha<0$. Hence, we can consider the function $(1+\ln n)^{\alpha} n^{\frac{\varepsilon}{2}} \uparrow$, and from the inequality (24), we obtain the following inequality:

$$
\begin{aligned}
& \sum_{n=1}^{\infty}\left(\rho_{n}^{r}-\rho_{n+1}^{r}\right) n^{\frac{r(q-2)}{2 q}}(1+\ln n)^{\alpha r} \\
& \quad \leq C \sum_{k=2}^{\infty}\left(1+\ln \left(n_{k}-1\right)^{\alpha r}\left(n_{k}-1\right)^{\frac{r}{2} \varepsilon}\right) \sum_{n=n_{k}-1}^{n_{k+1}-1}\left|a_{n}\right|^{r} n^{r\left(1-\frac{1}{q}\right)-1} \\
& \quad \leq C \sum_{k=2}^{\infty} \sum_{n=n_{k}-1}^{n_{k+1}-1}\left|a_{n}\right|^{r} n^{r\left(1-\frac{1}{q}\right)-1}(1+\ln n)^{\alpha r}
\end{aligned}
$$

for the case of $2<r<\infty, \alpha<0$. Thus, it follows from inequalities (30), (31) and (34) that

$$
\sum_{n=1}^{\infty}\left(\rho_{n}^{r}-\rho_{n+1}^{r}\right) n^{\frac{r(q-2)}{2 q}}(1+\ln n)^{\alpha r} \leq C \sum_{n=1}^{\infty}\left|a_{n}\right|^{r} n^{r\left(1-\frac{1}{q}\right)-1}(1+\ln n)^{\alpha r}
$$

and the proof is complete.

Our next result reads as follows.

Proposition 4.2 Let $\left\{\varphi_{n}\right\}_{n=1}^{\infty}$ be an uniformly bounded orthogonal system, $2<q<+\infty, \alpha \in$ $\mathbb{R}$ and $r>0$. If $\left|a_{n}\right| \downarrow 0, n \rightarrow \infty,\left\{a_{n}\right\} \in l_{2}$ and

$$
\sum_{n=1}^{\infty}\left|a_{n}\right|^{r} n^{r\left(1-\frac{1}{q}\right)-1}(1+\ln n)^{\alpha r}<+\infty
$$

then the series $\sum_{n=1}^{\infty} a_{n} \varphi_{n}(x)$ converges to some function $f \in L_{q, r}(\log L)^{\alpha}$ and

$$
\|f\|_{q, r, \alpha} \leq C\left\{\sum_{n=1}^{\infty}\left|a_{n}\right|^{r} n^{r\left(1-\frac{1}{q}\right)-1}(1+\ln n)^{\alpha r}\right\}^{\frac{1}{r}} .
$$

Proof It is easy to see that

$$
\begin{aligned}
& \sum_{n=1}^{\infty}\left(\rho_{n}^{r}-\rho_{n+1}^{r}\right) n^{\frac{r(q-2)}{2 q}}(1+\log n)^{\alpha r} \\
& \quad \leq C \sum_{k=1}^{\infty} 2^{k^{\frac{r(q-2)}{2 q}}}(1+k)^{\alpha r}\left(\rho_{2^{k-1}}^{r}-\rho_{2^{k}}^{r}\right) \\
& \quad=C \sum_{k=1}^{\infty} 2^{k \frac{r(q-2)}{2 q}}(1+k)^{\alpha r}\left(\sum_{v=k}^{\infty} \sum_{l=2^{v-1}}^{2^{v}-1}\left|a_{l}\right|^{2}\right)^{\frac{r}{2}} .
\end{aligned}
$$


Since $2<q<\infty$, we have

$$
\sum_{k=1}^{v} 2^{k \frac{r(q-2)}{2 q}}(1+k)^{\alpha r} \leq C 2^{\nu \frac{r(q-2)}{2 q}}(1+v)^{\alpha r}, \quad v=1,2 \ldots
$$

Therefore, by Lemma E, we obtain

$$
\begin{gathered}
\sum_{k=1}^{\infty} 2^{k \frac{r(q-2)}{2 q}}(1+k)^{\alpha r}\left(\sum_{\nu=k}^{\infty} \sum_{l=2^{\nu-1}}^{2^{\nu}-1}\left|a_{l}\right|^{2}\right)^{\frac{r}{2}} \\
\leq \sum_{k=1}^{\infty} 2^{k \frac{r(q-2)}{2 q}}(1+k)^{\alpha r}\left(\sum_{l=2^{k-1}}^{2^{k}-1}\left|a_{l}\right|^{2}\right)^{\frac{r}{2}} .
\end{gathered}
$$

Moreover, since $\left|a_{n}\right| \downarrow 0, n \rightarrow \infty$, it yields

$$
\left(\sum_{l=2^{k-1}}^{2^{k}-1}\left|a_{l}\right|^{2}\right)^{\frac{r}{2}} \leq\left(2^{k-1}\right)^{\frac{r}{2}}\left|a_{2^{k-1}}\right|^{r}, \quad k=1,2, \ldots
$$

Thus,

$$
\sum_{k=1}^{\infty} 2^{k \frac{r(q-2)}{2 q}}(1+k)^{\alpha r}\left(\sum_{l=2^{k-1}}^{2^{k}-1}\left|a_{l}\right|^{2}\right)^{\frac{r}{2}} \leq C \sum_{k=1}^{\infty} 2^{k r\left(1-\frac{1}{q}\right)}(1+k)^{\alpha r}\left|a_{2^{k-1}}\right|^{r} .
$$

Furthermore, since the sequence $\left\{\left|a_{n}\right|\right\}$ is monotonic, we can easily verify that

$$
\sum_{n=2^{k-2}}^{2^{k-1}-1}\left|a_{n}\right|^{r} n^{r\left(1-\frac{1}{q}\right)-1}(1+n)^{\alpha r} \geq C\left|a_{2^{k-1}}\right|^{r} 2^{k r\left(1-\frac{1}{q}\right)}(1+k)^{\alpha r}, \quad k=2,3, \ldots
$$

Therefore, it follows from inequality (37) that

$$
\sum_{k=1}^{\infty} 2^{k \frac{r(q-2)}{2 q}}(1+k)^{\alpha r}\left(\sum_{l=2^{k-1}}^{2^{k}-1}\left|a_{l}\right|^{2}\right)^{\frac{r}{2}} \leq C\left\{\left|a_{1}\right|^{r}+\sum_{n=1}^{\infty}\left|a_{n}\right|^{r} n^{r\left(1-\frac{1}{q}\right)-1}(1+n)^{\alpha r}\right\} .
$$

Now, from the inequalities (35), (36), and (38) we can deduce that

$$
\sum_{n=1}^{\infty}\left(\rho_{n}^{r}-\rho_{n+1}^{r}\right) n^{\frac{r(q-2)}{2 q}}(1+\log n)^{\alpha r} \leq C \sum_{n=1}^{\infty}\left|a_{n}\right|^{r} n^{r\left(1-\frac{1}{q}\right)-1}(1+n)^{\alpha r} .
$$

Therefore, in view of Corollary 2.3, the statement in the proposition holds.

Remark 4.3 We may ask wether it is possible to generalize the results obtained in this paper to more general Lorentz-Zygmund type spaces by replacing the weight $(1+|\ln t|)^{\alpha r} t^{r / q-1}$ by a more general weight $\lambda(t)$. Of course, we must still have some control of the growth properties of the weight. Below we will just briefly describe one such a possibility namely the quasi-monotone weights, used in recent work of Kopezhanova and Persson (see $[11,12])$. 
Let $0<r<\infty, 0<\beta<\infty$ and $\lambda=\lambda(t)$ be a positive function defined on $(0, \infty)$. Consider all functions $f$ for which

$$
\|f\|_{\Lambda_{\beta}(\lambda)}:=\left\{\int_{0}^{1}\left(f^{*}(t) t \lambda\left(\frac{1}{t}\right)\right)^{\beta} \frac{d t}{t}\right\}^{\frac{1}{\beta}}<+\infty .
$$

Note that if $\lambda(y)=y^{1-\frac{1}{q}}(\log (2 y))^{\alpha}, \alpha \in \mathbb{R}$, then, for $t \in(0,1]$, the function $t \lambda\left(\frac{1}{t}\right)=t^{\frac{1}{q}}(1+$ $\left.\log \frac{1}{t}\right)^{\alpha}$. Therefore

$$
\|f\|_{\Lambda_{\beta}}=\left\{\int_{0}^{1}\left(f^{*}(t)\right)^{\beta} t^{\frac{\beta}{q}}\left(1+\log \frac{1}{t}\right)^{\alpha \beta} \frac{d t}{t}\right\}^{\frac{1}{\beta}}
$$

so that $\Lambda_{\beta}$ is just the Lorentz-Zygmund space $L_{q, \beta}(\log L)^{\alpha}$.

We consider the following classes of functions $B=\bigcup_{\delta>0} B_{\delta}$ and $A=\bigcup_{\delta>0} A_{\delta}$ :

$$
\begin{aligned}
& B_{\delta}=\left\{\lambda: \lambda(y) y^{-\frac{1}{2}-\delta} \uparrow \text { and } \lambda(y) y^{-1+\delta} \downarrow \text { on }[1, \infty)\right\}, \\
& A_{\delta}=\left\{\lambda: \lambda(y) y^{-\frac{1}{2}-\delta} \uparrow \text { and } \lambda(y) y^{-1+\delta} \downarrow \text { on }[1, \infty)\right\} .
\end{aligned}
$$

The following result was proved by Kopezhanova and Persson (see [11, Theorem 2] and [12, p. 45]).

Theorem $\mathbf{F}$ Let $0<\beta<\infty$, and assume that the orthonormal system $\Phi=\left\{\varphi_{k}\right\}_{k=1}^{\infty}$ is uniformly bounded.

(a) If $\lambda(t)$ belongs to the class $A$, then

$$
\left(\sum_{n=1}^{\infty}\left(a_{n}^{*} \lambda(n)\right)^{\beta} \frac{1}{n}\right)^{\frac{1}{\beta}} \leq c_{1}\|f\|_{\Lambda_{\beta}(\lambda)},
$$

where $\left\{a_{n}^{*}\right\}$ is the nonincreasing rearrangement of the sequence $\left\{\left|a_{k}\right|\right\}_{k=1}^{\infty}$ of Fourier coefficients off with respect to the system $\Phi$.

(b) If $\lambda(t)$ belongs to the class $B$ and $f \stackrel{\text { a.e. }}{=} \sum_{n=1}^{\infty} a_{n} \varphi_{n}$, then

$$
\|f\|_{\Lambda_{\beta}(\lambda)} \leq c_{2}\left(\sum_{n=1}^{\infty}\left(a_{n}^{*} \lambda(n)\right)^{\beta} \frac{1}{n}\right)^{\frac{1}{\beta}} .
$$

Here the constants $c_{1}$ and $c_{2}$ do not depend on $f$.

In the case of $\lambda(y)=y^{1-\frac{1}{q}}(\log (2 y))^{\alpha}, \alpha \in \mathbb{R}$, from part (b) of Theorem $\mathrm{F}$ we obtain the following assertion.

Corollary 4.4 Let $0<\beta<\infty$, and assume that the orthonormal system $\Phi=\left\{\varphi_{k}\right\}_{k=1}^{\infty}$ is bounded. If $2<q<\infty, 0<\beta<\infty$, and $\stackrel{\text { a.e. }}{=} \sum_{n=1}^{\infty} a_{n} \varphi_{n}$, then

$$
\|f\|_{q, \beta, \alpha} \leq C\left(\sum_{n=1}^{\infty}\left(a_{n}^{*}\right)^{\beta} n^{\beta\left(1-\frac{1}{q}\right)-1}(1+\ln n)^{\alpha \beta}\right)^{\frac{1}{\beta}} .
$$


Proof For the function $\lambda(y)=y^{1-\frac{1}{q}}(\log (2 y))^{\alpha} \in B$ there exists a number $\delta>0$ such that $\lambda(y) \in B_{\delta}$. If $\frac{1}{2}-\frac{1}{q}-\delta>0$, then $\lambda(y) y^{-\frac{1}{2}-\delta}=y^{\frac{1}{2}-\frac{1}{q}-\delta}(1+\log y)^{\alpha} \uparrow$ on the interval $[1, \infty)$. Hence $2<q<\infty$.

Further, the function $\lambda(y) y^{-1+\delta}=y^{\delta-\frac{1}{q}}(1+\log y)^{\alpha} \downarrow$ on the interval $[1, \infty)$ if $\delta-\frac{1}{q}<0$.

Thus, there is a number $\delta \in\left(0, \min \left\{\frac{1}{q}, \frac{1}{2}-\frac{1}{q}\right\}\right)$ such that the function $\lambda(y)=y^{1-\frac{1}{q}}(1+$ $\log y)^{\alpha} \in B_{\delta}$. Therefore, by using (39), we see that the statement holds.

Remark 4.5 Obviously, Proposition 4.2 is more general than Corollary 4.4. We also note that in the case when the sequence $\left\{a_{n}\right\}_{n=1}^{\infty}$ is non-negative and decreasing the assertions of Proposition 4.2 and Corollary 4.4 coincide.

Remark 4.6 In [12] (see Theorem 2.1, Theorem 2.3), theorems on the convergence of series of the Fourier coefficients of a function from the generalized Lorentz space $\Lambda_{\beta}(\lambda)$ with respect to regular systems are proved. It is known that a regular system is uniformly bounded (see [16, p. 117]). Therefore, the assertions of Theorem 2.1 and Theorem 3.1 of this paper do not follow from the results of [12]. Since $\|f\|_{s} \leq\|f\|_{\infty}$, for the functions $f \in L_{\infty}[0,1]$, if orthogonal system $\left\{\varphi_{n}\right\}$ satisfies the condition (1), then $\left\{\varphi_{n}\right\}$ is uniformly bounded.

\section{Appendix: Proof of Lemma E}

The proof of Lemma $E$ is a consequence of a well-known inequality of Leindler [17]. For the reader's convenience we present a proof which is similar to but simpler than that in the research report [18] by Johansson.

(i) If $0<p<1$, then

$$
\left(\sum_{k=0}^{n} b_{k}\right)^{p} \leq \sum_{k=0}^{n} b_{k}^{p}
$$

By using this inequality, changing the order of summation and taking into account the condition (3) we get

$$
\sum_{n=0}^{\infty} a_{n}\left(\sum_{k=0}^{n} b_{k}\right)^{p} \leq \sum_{n=0}^{\infty} a_{n} \sum_{k=0}^{n} b_{k}^{p}=\sum_{k=0}^{\infty} b_{k}^{p} \sum_{n=k}^{\infty} a_{n} \leq C \sum_{k=0}^{\infty} a_{k} b_{k}^{p}
$$

in the case $0<p<1$.

Let $1 \leq p<\infty$. The following inequalities are proved in [17]:

$$
\begin{aligned}
& \sum_{n=0}^{\infty} a_{n}\left(\sum_{k=0}^{n} b_{k}\right)^{p} \leq p^{p} \sum_{n=0}^{\infty} a_{n}^{1-p}\left(\sum_{k=n}^{\infty} a_{k}\right)^{p} b_{n}, \\
& \sum_{n=0}^{\infty} a_{n}\left(\sum_{k=n}^{\infty} b_{k}\right)^{p} \leq p^{p} \sum_{n=0}^{\infty} a_{n}^{1-p}\left(\sum_{k=0}^{n} a_{k}\right)^{p} b_{n} .
\end{aligned}
$$

Now it is easy to verify that condition (3) and inequality (40) imply statement (i) also in the case of $1 \leq p<\infty$. 
(ii) If $0<p<1$, then

$$
\left(\sum_{k=n}^{\infty} b_{k}\right)^{p} \leq \sum_{k=n}^{\infty} b_{k}^{p}
$$

Using this inequality, changing the order of summation and taking into account the condition (4), we obtain

$$
\sum_{n=0}^{\infty} a_{n}\left(\sum_{k=n}^{\infty} b_{k}\right)^{p} \leq \sum_{n=0}^{\infty} a_{n} \sum_{k=n}^{\infty} b_{k}^{p}=\sum_{k=0}^{\infty} b_{k}^{p} \sum_{n=0}^{k} a_{n} \leq C \sum_{k=0}^{\infty} a_{k} b_{k}^{p}
$$

in the case $0<p<1$.

If $1 \leq p<\infty$, then statement (ii) follows from (4) and (41).

\section{Acknowledgements}

We thank the referees and Professors Dag Lukkasson and Annette Meidell for some good advice which improved the final version of the paper. Moreover, the first author is grateful for the support of this work given by the Russian Academic Excellence Project (agreement no. 02.A03.21.0006 of August 27, 2013, between the Ministry of Education and Science of the Russian Federation and Ural Federal University).

Funding

Not applicable.

Availability of data and materials

Not applicable.

\section{Competing interests}

The authors declare that they have no competing interests.

\section{Authors' contributions}

The authors contributed equally to the writing of this paper. All authors approved the final version of the manuscript.

\section{Author details}

${ }^{1}$ Department of Fundamental Mathematics, L.N. Gumilyov Eurasian National University, Astana, Republic of Kazakhstan.

${ }^{2}$ Institute of Mathematics and Computer Science, Ural Federal University, Yekaterinburg, Russia. ${ }^{3}$ Department of

Computer Science and Computational Engineering Campus Narvik, The Artic University of Norway, Narvik, Norway.

\section{Publisher's Note}

Springer Nature remains neutral with regard to jurisdictional claims in published maps and institutional affiliations.

Received: 6 February 2019 Accepted: 30 May 2019 Published online: 13 June 2019

\section{References}

1. Sharpley, R.: Counterexamples for classical operators on Lorentz-Zygmund spaces. Stud. Math. 58, 141-158 (1980)

2. Nikol'ski, S.M.: Approximation of Classes of Functions of Several Variables and Embedding Theorems. Nauka, Moscow (1977)

3. Zygmund, A.: Trigonometric Series, vol. II. Izdat. "Mir", Moscow (1965)

4. Marcinkiewicz, J., Zygmund, A.: Some theorems on orthogonal systems. Fundam. Math. 28, 309-335 (1937)

5. Maslov, A.V.: Estimates of Hausdorff-Young type for Fourier coefficients. Vestnik Moscow Univ. Ser. I Mat. Mekh. 3 19-22 (1982) (Russian)

6. Kolyada, V.I.: Some generalizations of the Hardy-Littlewood-Paley theorem. Mat. Zametki 54(3), 48-71 (1993) (Translation in Math. Notes 51(3-4), 908-922 (1992))

7. Kirillov, S.A.: Norm estimates of functions in Lorentz spaces. Acta Sci. Math. 65(1-2), 189-201 (1999)

8. Flett, T.M.: On a theorem of Pitt. J. Lond. Math. Soc. 2(7), 376-384 (1973)

9. Stein, E.M.: Interpolation of linear operators. Trans. Am. Math. Soc. 83, 482-492 (1956)

10. Bochkarev, S.V.: The Hausdorf-Young-Riesz theorem in Lorentz spaces and multiplicative inequalities. Tr. Mat. Inst. Steklova 219, 103-114 (1997) (Translation in Proc. Steklov Inst. Math. 219(4), 96-107 (1997))

11. Kopezhanova, A.N., Persson, L.-E.: On summability of the Fourier coefficients in bounded orthonormal systems for functions from some Lorentz type spaces. Eurasian Math. J. 1(2), 76-85 (2010)

12. Kopezhanova, A.: Summability of Fourier transforms of functions from Lorentz spaces. Ph.D. thesis, Department of Engineering Sciences and Mathematics, Luleå University of Technology (2017)

13. Persson, L.-E.: Relation between summability of functions and their Fourier series. Acta Math. Acad. Sci. Hung. 27(3-4), 267-280 (1976) 
14. Krein, S.G., Petunin, Y.I., Semenov, E.M.: Interpolation of Linear Operators. Nauka, Moscow (1978)

15. Mustahaeva, V.M., Akishev, G.: On the Fourier coefficients in Lorentz-Zygmund space. In: Modern Methods of the Theory of Functions and Problems, Voronezh, pp. 155-156 (2013)

16. Nursultanov, E.D.: On the coefficients of multiple Fourier series from $L_{p}$-spaces. Izv. Ross. Akad. Nauk, Ser. Mat. 64(1), 95-122 (2000) (Russian) (Translation in Izv. Math. 64(1), 93-120 (2000))

17. Leindler, L.: Generalization of inequalities of Hardy and Littlewood. Acta Sci. Math. 31, 279-285 (1970)

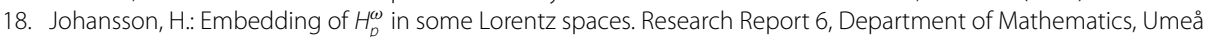
University, 36 pp. (1975)

Submit your manuscript to a SpringerOpen ${ }^{\circ}$ journal and benefit from:

- Convenient online submission

- Rigorous peer review

- Open access: articles freely available online

- High visibility within the field

- Retaining the copyright to your article

Submit your next manuscript at $\boldsymbol{\Delta}$ springeropen.com 